KU-AMP-96009

INS-Rep.-1157

September 1996

\title{
Inequivalent Quantizations and Holonomy Factor from the Path-Integral Approach
}

\author{
Shogo TANimura \\ Department of Applied Mathematics and Physics \\ Graduate School of Engineering \\ Kyoto University \\ Kyoto 606-01 \\ Japan \\ (e-mail: tanimura@kuamp.kyoto-u.ac.jp) \\ AND \\ IzUmi Tsutsui \\ Institute for Nuclear Study \\ University of Tokyo \\ Midori-cho, Tanashi-shi, Tokyo 188 \\ Japan \\ (e-mail: tsutsui@insth1.ins.u-tokyo.ac.jp)
}

\begin{abstract}
A path-integral quantization on a homogeneous space $G / H$ is proposed based on the guiding principle 'first lift to $G$ and then project to $G / H$ '. It is then shown that this principle gives a simple procedure to obtain the inequivalent quantizations (superselection sectors) along with the holonomy factor (induced gauge field) found earlier by algebraic approaches. We also prove that the resulting matrix-valued path-integral is physically equivalent to the scalar-valued pathintegral derived in the Dirac approach, and thereby present a unified viewpoint to discuss the basic features of quantizing on $G / H$ obtained in various approaches so far.
\end{abstract}




\section{Introduction}

Nearly a quarter of a century ago, Laidlaw and DeWitt [1] studied, generalizing Schulman's idea [2] (see also [3]), the path-integral quantization on a multiply connected configuration space $Q$, and established the, by now well-known, path-integral formula for a transition amplitude given by summing up paths with weight factors characterized by the homotopy class of the path. Subsequently, Dowker [4] reconsidered their argument using the concept of covering space to provide a convenient and geometric framework to deal with the paths of different homotopy classes. In this covering space construction one considers the universal covering space $\bar{Q}$ of $Q$, for which $Q=\bar{Q} / \Gamma$ with $\Gamma$ being the discrete group of isometries of $\bar{Q}$ which is isomorphic to the fundamental group of the space, $\pi_{1}(Q)$. Thus, if $\bar{q}_{0} \in \bar{Q}$ is a representative point then every other point in $\bar{Q}$ which reduces to a point $q \in Q$ under the covering projection $\pi: \bar{Q} \rightarrow Q$ can be written as $\bar{q}=\bar{q}_{0} \gamma$ using some $\gamma \in \Gamma$, that is, $\pi\left(\bar{q}_{0} \gamma\right)=q$ for any $\gamma \in \Gamma$. The path-integral formula for the propagator $K^{Q}\left(q^{\prime}, q ; T\right)$ on $Q$ put forward in this construction then takes the form,

$$
K^{Q}\left(q^{\prime}, q ; T\right)=\sum_{\gamma \in \Gamma} \rho(\gamma) K^{\bar{Q}}\left(\bar{q}^{\prime} \gamma, \bar{q} ; T\right)
$$

A salient feature of this formula (1.1) is that the propagator on the multiply connected space $Q$ is defined with the help of the propagator $K^{\bar{Q}}\left(\bar{q}^{\prime} \gamma, \bar{q} ; T\right)=\int_{\bar{q}}^{\bar{q}^{\prime} \gamma} \mathcal{D} \bar{q} e^{i S_{\bar{Q}}(\bar{q})}$ on the covering space $\bar{Q}$, where $\bar{q}$ and $\bar{q}^{\prime}$ are chosen such that $\pi(\bar{q})=q$ and $\pi\left(\bar{q}^{\prime}\right)=q^{\prime}$. Another important point to note is that the weight factors $\rho(\gamma)$ form unitary irreducible representations of the fundamental group ${ }^{1} \pi_{1}(Q)[1,4]$. One therefore finds many inequivalent quantizations (superselection sectors) depending on which representation of $\Gamma$ one uses for $\rho(\gamma)$. In the standard 'scalar quantum mechanics' where one uses scalar-valued (onecomponent) wave functions, the unitary representation must be one-dimensional, whereas it becomes multi-dimensional when, due to some internal symmetries, vector-valued (multicomponent) wave functions are considered [5].

Meanwhile, even prior to the above investigations, quantization on a configuration space of the form $Q=G / H$, where $G$ is a Lie group and $H$ its subgroup, was initiated by Mackey [6] (see also [7]) based on the imprimitivity relations, which is a generalization of the canonical commutation relations (Weyl relations). It was then found that such a homogeneous space $Q=G / H$ admits inequivalent quantizations which are labelled by the irreducible representations of $H$. Moreover, it was uncovered recently [8] in investigating the dynamical consequence of these inequivalent quantizations that different superselection

\footnotetext{
1 More precisely, the weight factors form unitary representations of the homology group $H_{1}(Q, \mathbb{Z})$, i.e., the 'Abelianized' $\pi_{1}(Q)$; see [5].
} 
sectors come equipped with a specific type of induced gauge field $A(q)$, called the canonical connection (or the $H$-connection) on the homogeneous space $G / H$, which is a solution of the Yang-Mills equations on $G / H[9,10]$. (A similar result has been obtained for the case $Q=S^{n}$ in [11].) The approaches employed in the above analyses are based purely on algebraic constructions, but it is worthwhile to mention that the resulting matrix-valued path-integral is characterized geometrically by the holonomy factor $\mathcal{P} \exp \left(-\int_{0}^{T} d t A\right)$ associated with the induced connection. Interestingly, one can also show that the propagator on $G / H$ admits a formula similar to (1.1) where now $\bar{Q}$ and $\Gamma$ are replaced by $G$ and $H$, respectively [8].

In view of the similarity in the outcome of quantization between the two types of configuration spaces, $Q=\bar{Q} / \Gamma$ and $Q=G / H$, we are tempted to reverse the logic and set up a quantization procedure for the latter type of spaces purely by the path-integral approach. In other words, we are interested in deriving all the known results obtained by algebraic approaches for a homogeneous space $Q=G / H$ starting from a path-integral formula defined along the line of $[1,3,4]$ for a multiply connected space $Q=\bar{Q} / \Gamma$. The aim of this paper is to carry out this programme, by completing our earlier attempt made in [12]. Indeed, we shall show that it is not only possible but also quite simple to do this, once we follow a geometrically motivated guiding principle which underlies the covering space construction of the path-integral for a multiply connected space. Note however that for $Q=\bar{Q} / \Gamma$ the inequivalent quantizations arise only if the fundamental group $\pi_{1}(Q)$ of the space is nontrivial whereas for $Q=G / H$ they arise even if it is trivial $\left(\pi_{1}(Q)=0\right)$, although for both types of spaces they are characterized by the irreducible representations of the subgroup $\Gamma$ or $H$. Thus the path-integral approach that we are going to discuss may be regarded as a generalization of the approach proposed for the quantization on $Q=\bar{Q} / \Gamma$, but the important point is that this generalization is necessary to reproduce the known results of quantization on $G / H$. In fact, such a generalization was mentioned in [4] but dropped on the ground that it might have modified the dynamics, which we now know that it should do, due to the induced connection.

Another approach to quantizing on $G / H$ was developed more recently in $[13,14]$, where the system of interest on $G / H$ is regarded as an effective constrained system on $G$ and quantized using the Dirac approach [15] to constrained systems. One of the advantages of this picture is that one obtains a scalar-valued path-integral (at the expense of introducing extra degrees of freedom) which is easier to deal with than the matrix-valued one in the previous approaches. We will show however that this scalar-valued path-integral can be derived from the matrix-valued path-integral using the technique of coherent state path-integrals [16], hoping that the path-integral approach presented here will furnish a 
unified viewpoint to discuss the various features of quantizing on $G / H$ obtained by different approaches so far.

The plan of the paper is as follows: In Section 2 we set up the procedures of the (generalized) path-integral quantization based on the principle mentioned above, and show that the path-integral leads correctly to the inequivalent quantizations possessing the holonomy factor (induced connection) in the required form. Then the equivalence of the two path-integrals, scalar-valued and matrix-valued, is proven in Section 3. Section 4 is devoted to the Conclusion and discussion, where a possible application and extension of the path-integral is also discussed. An appendix is provided to collect conventions used in the text.

\section{The path-integral on a homogeneous space $Q$}

In this section we wish to show that the path-integral on a homogeneous space $Q$ describing Mackey's inequivalent quantizations can readily be derived if we adopt the guiding principle that we first lift to $G$ and then project to $G / H$, where $G$ is a simply connected group for which $Q=G / H$, allowing a phase factor in the path-integral. This is basically the same idea used in the covering space construction for the propagator on a multiply connected space, but we first formulate it more precisely on a homogeneous space $G / H$, and then prove that the matrix-valued path-integral with the holonomy factor mentioned earlier is a direct consequence of our definition. The implications of the guiding principle will be discussed later.

\subsection{Definition}

In order to quantize a classical system whose configuration space $Q$ is a homogeneous space, we shall adopt, according to our guiding principle, the following three-step programme:

(i) Find a simply connected, semisimple group $G$ with which the configuration space is regarded as $Q=G / H$ with $H$ a closed subgroup of $G$.

(ii) Choose a classical system on $G$ which is invariant under the action of $H$ such that its classical reduction to $G / H$ gives the system on $Q$.

(iii) Define the path-integral on $Q=G / H$ by projecting the path-integral on $G$ down to $Q$, allowing a phase factor to appear.

To spell out the last step more explicitly, let us consider the propagator on the lifted system of $G$,

$$
K^{G}\left(g^{\prime}, g ; T\right)=\int_{g}^{g^{\prime}} \mathcal{D} g e^{i S_{G}(g)}
$$


which is invariant under the right translation with respect to the subgroup $H$,

$$
K^{G}\left(g^{\prime} h, g h ; T\right)=K^{G}\left(g^{\prime}, g ; T\right) .
$$

The measure $\mathcal{D} g$ in the path-integral (2.1) is formally a product of the (normalized) Haar measure $d g$ on $G$ which is (right) invariant, $d(g h)=d g$. Thus the invariance (2.2) is guaranteed if the action $S_{G}(g)$ of the system is invariant under (time independent) transformations $S_{G}(g h)=S_{G}(g)$. Equivalently, the potential in the action

$$
S_{G}(g)=\int_{0}^{T} d t\left(\frac{1}{2}\|\dot{g}\|^{2}-V(g)\right),
$$

which reduces to the potential on $Q$ upon projection, is assumed to be invariant $V(g h)=$ $V(g)$. In (2.3) the dot denotes the time derivative $\dot{g}=d g / d t$, and the norm $\|\cdot\|$ is given by the invariant metric on $G$, that is, $\|\dot{g}\|^{2}:=\operatorname{tr}\left(g^{-1} \dot{g}\right)^{2}$ with 'tr' being a matrix trace properly normalized in some irreducible representation (for our conventions used in this paper, see Appendix).

To furnish necessary geometry to implement the lift and projection step, we recall that, with $G / H$ viewed as the left cosets $\{g H \mid g \in G\}$, any element $g \in G$ admits the decomposition,

$$
g=\sigma(q) h,
$$

where $\sigma(q) \in G$ is a map $G / H \rightarrow G$ and $h \in H$. If we regard $G$ as a principal bundle $G(G / H, H)$ where $G / H$ is the base space and $H$ the fibre, then the map $\sigma(q)$ provides a section on the bundle. With this section the projection map $\pi: G \rightarrow G / H$ given by $\pi(g):=g H$ satisfies $\pi(\sigma(q))=q$, thus yielding $\pi(g)=q$ when the decomposition (2.4) is used. (Unless the bundle is trivial, the section $\sigma$ is defined only locally, and we need to introduce an open covering $\left\{D_{\alpha}\right\}$ of $Q=\cup_{\alpha} D_{\alpha}$ on which local sections $\left\{\sigma_{\alpha}: D_{\alpha} \rightarrow G\right\}$ are given. In this paper, however, for brevity we ignore the treatment necessary to deal with the locality of the sections; see [12].)

Given a path $q(t)$ in $Q$ with $q(0)=q$ and $q(T)=q^{\prime}$, the geometrical setting provided above allows us to lift it to the path $g(t)=\sigma(q(t)) h(t)$ in $G$ with $h(0)=1$ and $h(T)=h$. We can then consider the propagation from $q$ to $q^{\prime}$ on $Q=G / H$ by the corresponding lifted propagation from $\sigma(q)$ to $\sigma\left(q^{\prime}\right) h$ on $G$, where $h \in H$ represents the redundancy along the fibre (we need to consider the redundancy only at the final point $t=T$ because of the invariance (2.2)). Thus, with the (normalized) Haar measure $d h$ on $H$ we define the path-integral on $G / H$ by the following form:

$$
K^{G / H}\left(q^{\prime}, q ; T\right):=\int_{H} d h \rho(h) K^{G}\left(\sigma\left(q^{\prime}\right) h, \sigma(q) ; T\right) .
$$


The integration over $h \in H$ is performed in order to implement the projection from $G$ to $Q$ by summing up the final points $\sigma\left(q^{\prime}\right) h$. The point to be noted here is that in this projection we allow an $h$-dependent weight factor $\rho(h)$ to appear, in analogy with the case of multiply connected spaces (1.1).

The weight factor is not entirely arbitrary but subject to the condition that the propagator in (2.5) must fulfill the composition law,

$$
K^{G / H}\left(q^{\prime}, q ; T\right)=\int_{Q} d q^{\prime \prime} K^{G / H}\left(q^{\prime}, q^{\prime \prime} ; T-t\right) K^{G / H}\left(q^{\prime \prime}, q ; t\right),
$$

where $d q^{\prime \prime}$ is the $G$-invariant measure on $Q=G / H$ induced from the measure $d g$ through the decomposition (2.4), i.e.,

$$
\int_{G} d g f(g)=\int_{Q} d q \int_{H} d h f(\sigma(q) h),
$$

for a function $f(g)$ on $G$. Using the composition law for the propagator $K^{G}\left(g^{\prime}, g ; T\right)$ on $G$, one finds that (2.6) holds if

$$
\rho(h) \rho\left(h^{\prime}\right)=\rho\left(h h^{\prime}\right), \quad \text { for } \quad h, h^{\prime} \in H .
$$

This property (2.8) implies that the weight factor furnishes a representation of the group $H$. Thus, unless the representation is trivial and one-dimensional, the wave functions on which the propagator (2.5) acts are vector-valued, and the Hilbert space to which these wave functions belong is specified by the representation used. For definiteness, we consider for $\rho(h)$ a highest weight representation labeled by the highest weight $\chi$ (see Appendix),

$$
\rho(h)=\rho_{\mu \nu}^{\chi}(h):=\langle\chi, \mu|h| \chi, \nu\rangle .
$$

The propagator (2.5) is hence matrix-valued, $K^{G / H}\left(q^{\prime}, q ; T\right)=K_{\mu \nu}^{G / H}\left(q^{\prime}, q ; T\right)$, with indices given by the weight vectors belonging to the representation $\chi$. The wave functions we consider thus take the values in the linear space $V_{\chi}$ of the highest weight representation $\chi$. The Peter-Weyl theorem then states that the set of the weight factors (2.9) provides a complete set of basis functions for the wave functions on the group manifold $H$, for which the orthonormality relations read

$$
\int_{H} d h \rho_{\mu \nu}^{\chi}(h) \rho_{\mu^{\prime} \nu^{\prime}}^{\chi^{\prime} *}(h)=\frac{1}{d_{\chi}} \delta^{\chi \chi^{\prime}} \delta_{\mu \mu^{\prime}} \delta_{\nu \nu^{\prime}},
$$

where $d_{\chi}=\operatorname{dim} V_{\chi}$ is the dimension of the representation $\chi$. (Incidentally, we mention that the propagator (2.5) depends on the sections used. For example, if the initial point 
$q$ lies in the covering $D_{\alpha}$ whereas the final point $q^{\prime}$ lies in the overlap $D_{\beta} \cap D_{\gamma}$ of the two coverings, $D_{\beta}$ and $D_{\gamma}$, then the propagator $K_{\mu \nu}^{G / H}\left(q^{\prime}, q ; T\right)^{(\gamma \alpha)}$ defined using $\sigma_{\gamma}\left(q^{\prime}\right)$ and $\sigma_{\alpha}(q)$ is equal to $\rho_{\mu \lambda}^{\chi}\left(\sigma_{\gamma}^{-1}\left(q^{\prime}\right) \sigma_{\beta}\left(q^{\prime}\right)\right) K_{\lambda \nu}^{G / H}\left(q^{\prime}, q ; T\right)^{(\beta \alpha)}$ where the latter propagator is defined using $\sigma_{\beta}\left(q^{\prime}\right)$ and $\sigma_{\alpha}(q)$.)

We note in passing that, if the propagator on $G$ fulfills the standard normalization condition, $\lim _{T \rightarrow 0} K^{G}\left(g^{\prime}, g ; T\right)=\delta_{G}\left(g, g^{\prime}\right)$, then the propagator on $G / H$ defined in (2.5) satisfies

$$
\lim _{T \rightarrow 0} K_{\mu \nu}^{G / H}\left(q^{\prime}, q ; T\right)=\delta_{\mu \nu} \delta_{Q}\left(q, q^{\prime}\right),
$$

where $\delta_{G}\left(g, g^{\prime}\right)$ and $\delta_{Q}\left(q, q^{\prime}\right)$ are delta-functions on $G$ and $Q=G / H$, respectively.

In conclusion, we see that our guiding principle leads to the path-integral formula (2.5) for a homogeneous space possessing weight factors given by a unitary representation of the isometry group $H$. The significance of the obvious similarity between the two path-integral formulae, (2.5) and (1.1), will be discussed in Section 4.

\subsection{The holonomy factor}

Now we are in a position to derive the path-integral on $G / H$ possessing the holonomy factor from the definition (2.5) which, from what we have found, becomes

$$
K_{\mu \nu}^{G / H}\left(q^{\prime}, q ; T\right)=\int_{H} d h \rho_{\mu \nu}^{\chi}(h) \int_{q}^{q^{\prime}} \mathcal{D} q \int_{e}^{h} \mathcal{D} h e^{i S_{G}(\sigma(q) h)} .
$$

Here we decomposed the path-integral measure $\mathcal{D} g=\mathcal{D} q \mathcal{D} h$ based on the decomposition (2.4) intending to integrate out the $H$ degrees of freedom to get an expression purely in terms of the path-integration on $G / H$. The decomposition (2.4) also induces the decomposition of the action as

$$
S_{G}(\sigma(q) h)=S_{G / H}(q)+S^{\prime}(q, h) .
$$

The first term is the action on $G / H$,

$$
S_{G / H}(q)=\int_{0}^{T} d t\left(\frac{1}{2}\|\dot{q}\|^{2}-V(\sigma(q))\right),
$$

which possesses the usual kinetic term of the particle moving under the induced metric of $G / H$ given by the norm $\|\cdot\|$ in $G / H$, i.e., $\|\dot{q}\|^{2}:=\operatorname{tr}\left(\left.\sigma^{-1}(q) \dot{\sigma}(q)\right|_{\mathfrak{r}}\right)^{2}$ (we denote the decomposition of an element $X$ of the Lie algebra $\mathfrak{g}$ of $G$ as $X=\left.X\right|_{\mathfrak{h}}+\left.X\right|_{\mathfrak{r}}$, where $\mathfrak{h}$ is the Lie algebra of $H$ while $\mathfrak{r}$ is the orthogonal complement; see Appendix). On the other hand, the second term in (2.13),

$$
S^{\prime}(q, h)=\int_{0}^{T} d t \frac{1}{2} \operatorname{tr}\left(\dot{h} h^{-1}+A(q)\right)^{2},
$$


contains the kinetic term for $h$ and the canonical connection $A(q):=\left.\sigma^{-1}(q) \dot{\sigma}(q)\right|_{\mathfrak{h}}$ mentioned earlier.

We here observe that, if we change the variables in the path-integral measure $\mathcal{D} h$ in $(2.12)$ as $h(t)_{\text {old }} \rightarrow h(t)_{\text {new }}:=\tilde{h}^{-1}(t) h(t)_{\text {old }}$ for $0<t<T$ in such a way that the function $\tilde{h}(t)$ obeys the differential equation

$$
\dot{\tilde{h}} \tilde{h}^{-1}+A(q)=0
$$

then we can eliminate the canonical connection $A(q)$ from the action, thereby reducing $S^{\prime}(q, h)$ to the action for the free particle on $H$,

$$
S_{H}(h)=\int_{0}^{T} d t \frac{1}{2}\|\dot{h}\|^{2}
$$

This, however, does not imply that the $q$-dependence disappears entirely from the final result, because the change of variables causes a shift in the boundary values of the pathintegral in (2.12) as $\int_{e}^{h} \mathcal{D} h \rightarrow \int_{\tilde{h}^{-1}(0)}^{\tilde{h}^{-1}(T) h} \mathcal{D} h$. But this $q$-dependence in the boundary values can also be eliminated by changing the variable in the integration $d h$ in (2.12) analogously as $h_{\text {old }} \rightarrow h_{\text {new }}:=\tilde{h}^{-1}(T) h_{\text {old }}$. Choosing the initial condition $\tilde{h}(0)=e$ for the solution of the equation (2.16), we find after these successive changes of variables that the pathintegral is decomposed into two parts; one over $G / H$ and the other over $H$,

$$
K_{\mu \nu}^{G / H}\left(q^{\prime}, q ; T\right)=\int_{q}^{q^{\prime}} \mathcal{D} q \rho_{\mu \eta}^{\chi}(\tilde{h}(T)) e^{i S_{G / H}(q)} \cdot \int_{H} d h \rho_{\eta \nu}^{\chi}(h) \int_{e}^{h} \mathcal{D} h e^{i S_{H}(h)} .
$$

Let us first evaluate the second part in (2.18). Note that the Hamiltonian $\widehat{H}$ describing the free particle on the group manifold $H$ corresponding to (2.17) is just (half) the quadratic Casimir operator whose orthonormal eigenfunctions are given by the set $\left\{\sqrt{d_{\chi}} \rho_{\mu \nu}^{\chi^{*}}(h)\right\}$ in (2.9). Thus if we recall the standard formula that the path-integral $\int_{x}^{x^{\prime}} \mathcal{D} x e^{i S(x)}$ can be expressed as $\left\langle x^{\prime}\left|e^{-i \widehat{H} T}\right| x\right\rangle=\sum_{n} u_{n}\left(x^{\prime}\right) e^{-i E_{n} T} u_{n}^{*}(x)$ in terms of the eigenfunctions $u_{n}(x)=\langle x \mid n\rangle$ of energy $E_{n}$, we find that the path-integral on $H$ in the second part reads

$$
\int_{e}^{h} \mathcal{D} h e^{i S_{H}(h)}=\sum_{\chi, \mu, \nu} d_{\chi} \rho_{\mu \nu}^{\chi *}(h) e^{-\frac{i}{2} C_{2}^{\chi} T} \rho_{\mu \nu}^{\chi}(e)
$$

where $C_{2}^{\chi}$ is the value of the Casimir on the eigenfunction $\sqrt{d_{\chi}} \rho_{\mu \nu}^{\chi^{*}}(h)$. Then, with the orthonormality relations $(2.10)$, the second part is evaluated to be

$$
\int_{H} d h \rho_{\eta \nu}^{\chi}(h) \sum_{\chi^{\prime}, \mu^{\prime}} d_{\chi^{\prime}} \rho_{\mu^{\prime} \mu^{\prime}}^{\chi^{\prime}}(h) e^{-\frac{i}{2} C_{2}^{\chi^{\prime} T}}=e^{-\frac{i}{2} C_{2}^{\chi} T} \delta_{\eta \nu}
$$


For the first part in (2.18) we only need to note that the solution of (2.16) with the required initial condition is given by the path-ordered product of the canonical connection, i.e., the holonomy factor,

$$
\tilde{h}(T)=\mathcal{P} e^{-\int_{0}^{T} d t A} .
$$

Substituting (2.20) and (2.21) into (2.18), we finally obtain the desired expression,

$$
K_{\mu \nu}^{G / H}\left(q^{\prime}, q ; T\right)=e^{-\frac{i}{2} C_{2}^{\chi} T} \int_{q}^{q^{\prime}} \mathcal{D} q \rho_{\mu \nu}^{\chi}\left(\mathcal{P} e^{-\int_{0}^{T} d t A}\right) e^{i S_{G / H}(q)},
$$

which is the path-integral derived in [8] based on the Trotter formula. (The expression (2.22) has also been derived in our previous paper [12] using a stationary point approximation; but here we have presented a better derivation without any approximation.)

\subsection{Implications of the guiding principle}

Having derived the path-integral formula on $Q=G / H$ in the desired form (2.22), we now return to the path-integral (2.5) which we started with and discuss what it actually implies. For this purpose, it is instructive to consider the simple example $Q=S^{1}$. According to the guiding principle, one has to lift it to a group manifold which is simply connected ( $S^{1}$ is already a group manifold $S^{1}=U(1)$ but it is not simply connected). An obvious candidate is its universal covering space $\mathbb{R}$ with which $S^{1}=\mathbb{R} / \mathbb{Z}$, where now $\mathbb{R}$ is regarded as the manifold of the group of translations on a line. Then the above argument leading to the path-integral (2.22) applies formally to this case, even though the isometry group is discrete $H=\mathbb{Z}$ and hence one needs to replace, e.g., the integration $\int d h$ by the summation $\sum_{n \in \mathbb{Z}}$. But actually one does not need to go through the whole procedure to get the holonomy factor in (2.22), since the simplicity of the system allows for a direct derivation of the result almost from the outset once we follow the definition, that is, the path-integral on $Q=S^{1}$ be given by

$$
K^{S^{1}}\left(q^{\prime}, q ; T\right)=\sum_{n=-\infty}^{\infty} \rho(n) K^{\mathbb{R}}\left(q^{\prime}+2 \pi n, q ; T\right) .
$$

We have here used $q \in S^{1}=[0,2 \pi)$ and assumed that the propagator on a line is invariant $K^{\mathbb{R}}\left(x^{\prime}+2 \pi, x+2 \pi ; T\right)=K^{\mathbb{R}}\left(x^{\prime}, x ; T\right)$ for $x \in \mathbb{R}$ under the translation of the group $H=\mathbb{Z}$, which is ensured if the potential is periodic, $V(x+2 \pi)=V(x)$.

From the point of view of $S^{1}$, it is clear that the summation over the final points $q^{\prime}+2 \pi n$ in (2.23) amounts to performing the summation over all possible winding numbers $n$ that the paths on $S^{1}$ can take during the transition from $q$ to $q^{\prime}$ on the circle. The weight factor, which furnishes the representations of $\mathbb{Z}$, takes the form $\rho(n)=e^{i n \theta}$ where 
$\theta \in[0,2 \pi)$ is the angle parameter specifying the representation of $\mathbb{Z}$. Then, with $A:=$ $\theta /(2 \pi)$, it is easy to rewrite the propagator $(2.23)$ into the form,

$$
K^{S^{1}}\left(q^{\prime}, q ; T\right)=e^{-i \frac{\theta}{2 \pi}\left(q^{\prime}-q\right)} \sum_{n=-\infty}^{\infty} \int_{q}^{q^{\prime}+2 \pi n} \mathcal{D} q \exp \left[i \int d t\left\{\frac{1}{2} \dot{q}^{2}-V(q)+A \dot{q}\right\}\right] .
$$

We therefore see that the insertion of the weight factor in this case $Q=S^{1}$ provides in effect the minimal coupling with the vector potential $A$. Being constant, the vector potential has a vanishing curvature on $S^{1}$ but the flux penetrating the circle is finite and proportional to the parameter $\theta$. Hence, its physical consequence is analogous to that of the Aharonov-Bohm effect [17].

The appearance of the induced gauge potential $A$ is hence well illustrated by the example $Q=S^{1}=\mathbb{R} / \mathbb{Z}$, but unfortunately another important element which in general appears in the path-integral on $Q=G / H$ is missing due to its simplicity. The element missing is the matrix-valuedness of the propagator, which means that the Hilbert space consists of vector-valued wave functions taking values in $V_{\chi}$. This implies that in addition to the degrees of freedom represented by the position of the particle on $G / H$, we have some finite degrees of freedom represented by the vectors, which we may regard as a generalized spin since they reduce to the conventional spin for $H=S U(2)$. (The generalized spin does not arise for $S^{1}=\mathbb{R} / \mathbb{Z}$ since the subgroup $\mathbb{Z}$ has only one dimensional irreducible representations.) These extra degrees of freedom are in fact necessary in summing up paths connecting $\sigma(q)$ and $\sigma\left(q^{\prime}\right) h$ in which the particle can move along the fibre $H$ as well in the base space $G / H$. Since we have the kinetic energy term in $S_{H}$ in (2.17) (which is absent for $H=\mathbb{Z}$ ), in the generic case there exists a contribution from the integration along $H$.

The above feature seen in the formula (2.5) may somewhat be counter-intuitive as a path-integral over the base space $G / H$, but there is another, clearer characterization of the formula (2.5) as follows. Suppose we quantize a system on $G$ which is invariant under the action of $H$. The symmetry then allows for considering subspaces of the Hilbert space $L_{2}(G)$, which are classified according to the irreducible representations $\chi$ of the symmetry group $H$. The wave functions $\varphi(g)$ belonging to a subspace $\Gamma^{\chi}(G) \subset L_{2}(G)$ labeled by $\chi$ are hence $V_{\chi}$-valued and characterized by the $\chi$-equivariance under the $H$-action, $\varphi(g h)=\rho^{\chi}\left(h^{-1}\right) \varphi(g)$. Thanks to the symmetry, the propagation within the subspace $\Gamma^{\chi}(G)$,

$$
\left(U^{G}(T) \varphi\right)_{\mu}\left(g^{\prime}\right):=\int d g K^{G}\left(g^{\prime}, g ; T\right) \varphi_{\mu}(g),
$$

is well-defined, i.e., it furnishes a map $U^{G}(T): \Gamma^{\chi}(G) \rightarrow \Gamma^{\chi}(G)$. Now, using (the pullback of) some section $\sigma(q)$, we may define an isomorphism $\Pi$ from $\Gamma^{\chi}(G)$ to the space of $V_{\chi^{-}}$ valued functions on $G / H$, denoted by $\Gamma^{\chi}(G / H)$, and its inverse $\Pi^{-1}$ by

$$
\Pi: \psi_{\mu}(q):=\varphi_{\mu}(\sigma(q)), \quad \Pi^{-1}: \varphi_{\mu}(g):=\rho_{\mu \nu}^{\chi}\left(g^{-1} \sigma(\pi(g))\right) \psi_{\nu}(\pi(g)),
$$


for $\varphi \in \Gamma^{\chi}(G)$ and $\psi \in \Gamma^{\chi}(G / H)$. Then, the propagation on $\Gamma^{\chi}(G / H)$,

$$
\left(U^{G / H}(T) \psi\right)_{\mu}\left(q^{\prime}\right):=\int d q K_{\mu \nu}^{G / H}\left(q^{\prime}, q ; T\right) \psi_{\nu}(q)
$$

may be defined with the help of the propagation on $\Gamma^{\chi}(G)$ as $U^{G / H}:=\Pi \circ U^{G} \circ \Pi^{-1}$. Explicitly, it reads

$$
\begin{aligned}
\left(U^{G / H}(T) \psi\right)_{\mu}\left(q^{\prime}\right) & =\int d g K^{G}\left(\sigma\left(q^{\prime}\right), g\right) \rho_{\mu \nu}^{\chi}\left(g^{-1} \sigma(\pi(g))\right) \psi_{\nu}(\pi(g)) \\
& =\int d q d h \rho_{\mu \nu}^{\chi}\left(h^{-1}\right) K^{G}\left(\sigma\left(q^{\prime}\right) h^{-1}, \sigma(q)\right) \psi_{\nu}(q)
\end{aligned}
$$

where use has been made of the invariance (2.2). Comparing this to (2.27) and noting the invariance $d h^{-1}=d h$ of the Haar measure, we find that the propagator $K^{G / H}\left(q^{\prime}, q ; T\right)$ so defined reproduces the formula (2.5). This shows that the three step programme of quantization on $G / H$ stipulated by the guiding principle is actually equivalent to the above procedure involving the projection from the space of the equivariant functions $\Gamma^{\chi}(G)$ to the space $\Gamma^{\chi}(G / H)$. The formula $(2.5)$ provides a concise expression of this procedure in the path-integral framework.

\section{Description by the scalar-valued path-integral}

The path-integral on a homogeneous space $Q$ derived in Section 2 is generically matrixvalued. However, in ref.[14] it was shown that one can also describe Mackey's quantization using a scalar-valued path-integral. The key observation in [14] is that it is possible to realize the generalized spin taking its value in $V_{\chi}$ as the quantized version of the classical system of a coadjoint orbit of the group $H$. In this section we provide a direct proof that the two path-integrals, matrix-valued and scalar-valued, are different expressions of the same quantization on $Q$.

\subsection{Scalar-valued vs vector-valued wave functions}

Before going into the proof, we recall first the description of Mackey's inequivalent quantizations in terms of scalar-valued wave functions [14]. The wave functions that Mackey considered are vector-valued functions $\psi_{\mu}(q) \in \Gamma^{\chi}(G / H)$ characterized by the induced representation,

$$
\left(U_{\mathrm{L}}(g) \psi\right)_{\mu}(q)=\sum_{\nu} \rho_{\mu \nu}^{\chi}\left(\sigma^{-1}(q) g \sigma\left(g^{-1} q\right)\right) \psi_{\nu}\left(g^{-1} q\right)
$$


under the left $G$-action $q \rightarrow g^{-1} q$ on $G / H$. Our idea of constructing physically equivalent but scalar-valued wave functions is that we start by scalar-valued wave functions on $G$ and then find a suitable reduction to wave functions on $G / H$ in such a way that the degrees of freedom of $G$ be reduced to those of $G / H$ plus the generalized spin. The wave functions on $G$ are assumed to transform under the $G$-action by the usual (left and right) regular representations,

$$
\left(U_{\mathrm{L}}(\tilde{g}) \psi\right)(g)=\psi\left(\tilde{g}^{-1} g\right), \quad\left(U_{\mathrm{R}}(\tilde{g}) \psi\right)(g)=\psi(g \tilde{g})
$$

for $\tilde{g} \in G$. Thus the infinitesimal generators $\widehat{L}_{m}:=\operatorname{tr}\left(\widehat{L} T_{m}\right), \widehat{R}_{m}:=\operatorname{tr}\left(\widehat{R} T_{m}\right)$ associated with these left and right $G$-action corresponding to the basis $\left\{T_{m}\right\}$ of the Lie algebra $\mathfrak{g}$ of $G$ fulfill the commutation relations,

$$
\left[\widehat{R}_{m}, \widehat{R}_{n}\right]=i f_{m n}^{l} \widehat{R}_{l}, \quad\left[\widehat{L}_{m}, \widehat{L}_{n}\right]=i f_{m n}^{l} \widehat{L}_{l}, \quad\left[\widehat{R}_{m}, \widehat{L}_{n}\right]=0
$$

where $f_{m n}^{l}$ are structure constants of the algebra $\mathfrak{g}$.

To carry out the reduction we employ Dirac's approach [15] to quantizing a constrained system, where a set of constraints are imposed as 'physical state conditions' restricting the Hilbert space $L_{2}(G)$ to an appropriate subspace. To choose the constraints in our case, we consider the set of operators,

$$
\widehat{\phi}_{i}=\operatorname{tr}(\widehat{R}-K) T_{i} \quad \text { for } \quad T_{i} \in \mathfrak{h},
$$

where $\left\{T_{i}\right\}$ is a basis in the Lie algebra $\mathfrak{h}$ of the subgroup $H$, and $K \in \mathfrak{h}$ is a constant element which will be specified below. For $K=0$ the physical subspace, consisting of states satisfying $\hat{\phi}_{i} \psi_{\text {phy }}(g)=0$, reduces to the Hilbert space $L_{2}(G / H)$, because then the physical wave functions belonging to the subspace do not depend on the $H$ degrees of freedom $\psi_{\text {phy }}(g h)=\psi_{\text {phy }}(g)$ and can be regarded as wave functions on $Q=G / H$. For $K \neq 0$, however, we find from the commutator

$$
\left[\widehat{\phi}_{i}, \widehat{\phi}_{j}\right]=i f_{i j}^{k} \widehat{\phi}_{k}+i \operatorname{tr}\left(\left[T_{i}, T_{j}\right] K\right)
$$

that $\left\{\widehat{\phi}_{i}\right\}$ in (3.4) form a mixed (first and second class) set of operators and thus cannot be directly used to form a set of constraints to define the physical subspace.

To proceed, we have to first find a maximal set of first class components among the mixed set of operators, and to this purpose we consider the subalgebra $\mathfrak{s}_{K}:=\operatorname{Ker}\left(\operatorname{ad}_{K}\right) \cap \mathfrak{h}$ given by the kernel of the adjoint action of $K$ in $\mathfrak{h}$. For a generic $K$ (i.e., if $K$ is a regular semisimple element in $\mathfrak{h}$ ), the subalgebra $\mathfrak{s}_{K}$ is the Cartan subalgebra of $\mathfrak{h}$ containing $K$ 
[18] (for $K$ non-generic, see [14]). Choosing a basis $\left\{T_{r}\right\}$ in $\mathfrak{s}_{K}$, we see that for any $T_{j} \in \mathfrak{h}$ we have $\operatorname{tr}\left(\left[T_{r}, T_{j}\right] K\right)=0$ and hence the first class components in (3.4) are

$$
\widehat{\phi}_{r}:=\operatorname{tr} T_{r}(\widehat{R}-K) \quad \text { for } \quad T_{r} \in \mathfrak{s}_{K}
$$

Conversely, from the semisimplicity of $\mathfrak{h}$ it follows that these $\widehat{\phi}_{r}$ form the maximal set of the first class components in (3.4). We now need to find, from the operators in (3.4), the maximal subalgebra which can be used to define the physical subspace. For this, let us consider the complex extension $\mathfrak{h}_{\mathrm{c}}$ of the algebra $\mathfrak{h}$ and introduce a Chevalley basis $\left\{H_{\alpha_{r}}, E_{ \pm \varphi}\right\}$ in $\mathfrak{h}_{\mathrm{c}}$ (see Appendix) where we take $T_{r}:=\frac{1}{i} H_{\alpha_{r}}(r=1, \ldots, \operatorname{rank} H)$. Then the maximal subalgebra of (3.4) is given by the set $\left\{\widehat{\phi}_{r}, \widehat{\phi}_{\varphi}\right\}$, where

$$
\widehat{\phi}_{\varphi}=\operatorname{tr} E_{-\varphi}(\widehat{R}-K)=\operatorname{tr}\left(E_{-\varphi} \widehat{R}\right)
$$

for all positive roots $\varphi$. We then use these operators to define the physical states as

$$
\widehat{\phi}_{r} \psi_{\text {phy }}(g)=0 \quad \text { and } \quad \widehat{\phi}_{\varphi} \psi_{\text {phy }}(g)=0
$$

If the constants $K_{r}=\operatorname{tr}\left(T_{r} K\right)$ take the integer values ${ }^{2}$ corresponding to the highest weight $\chi$, i.e.,

$$
K_{r}=\chi\left(H_{\alpha_{r}}\right), \quad \text { for } \quad r=1, \ldots, \operatorname{rank} H
$$

then, on account of the property

$$
\widehat{R}_{r} \rho_{\mu \mu^{\prime}}^{\chi^{*}}(h)=\mu^{\prime}\left(H_{\alpha_{r}}\right) \rho_{\mu \mu^{\prime}}^{\chi^{*}}(h),
$$

the physical states defined by (3.8) have the solutions

$$
\psi_{\text {phy }}(g)=\psi_{\text {phy }}(q, h)=\sum_{\mu} \psi_{\mu}(q) \rho_{\mu \chi}^{\chi *}(h)
$$

The wave functions (3.11) provide a description of Mackey's quantization in terms of scalar wave-functions. Indeed, given a physical wave function (3.11), the coefficient functions in (3.11) are obtained as

$$
\psi_{\mu}(q)=d_{\chi} \int_{H} d h \psi_{\text {phy }}(q, h) \rho_{\mu \chi}^{\chi}(h),
$$

which reproduce exactly the induced representation (3.1) under the left-regular representation in (3.2) [14].

2 This integer-valuedness of the constant matrix $K$ can be derived from a consistency condition in the path-integral $[13,14]$; see below. 
Once the constraint point of view for Mackey's quantization is established, one can write down the effective path-integral implementing the constraints in the standard manner. Choosing gauge fixing conditions $\xi_{s}(g)=0$ for the gauge symmetry generated by the maximal set of first class components $(3.6)$, we find $[13,14]$ :

$$
K_{\text {eff }}\left(g^{\prime}, g ; T\right)=\mathcal{N} \int_{g}^{g^{\prime}} \mathcal{D} g \delta\left(\xi_{r}\right) \Delta_{\xi} e^{i S_{\text {eff }}}
$$

where $\mathcal{N}$ is a formal normalization constant and $\Delta_{\xi}:=\operatorname{det}\left|\left\{\phi_{r}, \xi_{r^{\prime}}\right\}\right|$ is the usual determinant factor necessary to render the path-integral gauge-independent. The effective action $S_{\text {eff }}$ appearing in the path-integral (3.13) consists of three parts $S_{\text {eff }}=S_{G / H}+S_{\text {coa }}+S_{\text {int }}$, where $S_{G / H}$ is the action on $G / H(2.14)$ whereas the other two are given by

$$
S_{\text {coa }}=-\int_{0}^{T} d t \operatorname{tr}\left(K h^{-1} \dot{h}\right), \quad S_{\text {int }}=-\int_{0}^{T} d t \operatorname{tr}\left(h K h^{-1} A(q)\right) .
$$

Here $S_{\text {coa }}$ is the action for the coadjoint orbit of $H$ passing through $K$, which is the system of generalized spin in the sense that variables parametrizing the coadjoint orbit $S:=$ $h K h^{-1}$ form the Lie algebra $\mathfrak{h}$ under Poisson bracket (or commutator after quantization) $[19,20]$. The action $S_{\text {int }}$ then describes the interation of the generalized spin with the canonical connection $A(q)$ in a way similar to Wong's particle coupled to a Yang-Mills field [21]. Finally we note that, for the path-integral (3.13) to be well-defined under the presence of the gauge symmetry, the constant matrix $K$ must take the integer values (3.9) $[13,14]$, and that this is consistent with the fact that quantization of coadjoint orbits leads to irreducible representations of the group.

\subsection{Scalar-valued path-integral}

We now show that the effective scalar-valued path-integral (3.13) found in the Dirac approach can directly be obtained from our matrix-valued path-integral with the holonomy factor (2.22). More explicitly, we shall evaluate the propagator $K_{\mathrm{phy}}^{G / H}\left(g^{\prime}, g ; T\right)$ for the scalar-valued physical wave-functions (3.11) defined in

$$
\left(U^{G / H}(T) \psi_{\text {phy }}\right)\left(g^{\prime}\right):=\int d g K_{\text {phy }}^{G / H}\left(g^{\prime}, g ; T\right) \psi_{\text {phy }}(g)
$$

and show that it coincides with the effective path-integral (3.13).

To this end, we first substitute the physical states in (3.15) with the solutions (3.11) and use the propagator (2.22) for the coefficent vector-valued wave functions to get

$$
K_{\text {phy }}^{G / H}\left(g^{\prime}, g ; T\right)=d_{\chi} e^{-\frac{i}{2} C_{2}^{\chi} T} \int_{q}^{q^{\prime}} \mathcal{D} q \rho_{\chi \chi}^{\chi}\left(h^{\prime-1} \mathcal{P} e^{-\int_{0}^{T} d t A} h\right) e^{i S_{G / H}(q)}
$$


What remains to do is to rewrite the holonomy factor in (3.16) in terms of a path-integral over $H$. This is achieved if we recall the construction of the generalized coherent state pathintegral of the group $H$ [16], where the key formula is the 'resolution of unity' identity,

$$
d_{\chi} \int_{H} d h h|\chi, \nu\rangle\langle\chi, \nu| h^{-1}=1
$$

This identity (3.17) can be derived from the orthonormality relations (2.10) by putting $\chi=\chi^{\prime}$ and $\nu=\nu^{\prime}$ using (2.9). Note that the integrand of (3.17) is constant along the Cartan subgroup $S_{K}:=e^{\mathfrak{s}_{K}}$ of $H$, and hence the integration over $S_{K}$ yields merely the volume $V_{S_{K}}=\int_{S_{K}} d h$ of the group $S_{K}$. Hence, if we employ the same trick used in the path-integral (3.13) to isolate the measure for $S_{K}$ in the measure $d h$ for $H$, we may write (3.17) as

$$
\int \widetilde{d h} h|\chi, \nu\rangle\langle\chi, \nu| h^{-1}=1
$$

where $\widetilde{d h}:=d_{\chi} V_{S_{K}} d h \delta\left(\xi_{r}\right) \Delta_{\xi}$ with $\xi_{r}(h)=0$ being conditions to fix the $S_{K}$ degrees of freedom, now regarded as functions of $H$.

To build up a coherent state path-integration, we break, as usual, the time interval $[0, T]$ into $N+1$ short slices of duration $\Delta t=\frac{T}{N+1}$ as $0=t_{0}<t_{1}<\cdots<t_{N}<t_{N+1}=T$. Inserting the identity (3.18) with $\nu=\chi$ at each slice of time in the holonomy factor in (3.16) and setting $h(0)=h$ and $h(T)=h^{\prime}$, we find

$$
\rho_{\chi \chi}^{\chi}\left(h^{\prime-1} \mathcal{P} e^{-\int_{0}^{T} d t A} h\right)=\int \prod_{n=1}^{N} \widetilde{d h}\left(t_{n}\right) \prod_{n=0}^{N}\left\langle\chi, \chi\left|h^{-1}\left(t_{n+1}\right) \mathcal{P} e^{-\int_{t_{n}}^{t_{n+1}} d t A(t)} h\left(t_{n}\right)\right| \chi, \chi\right\rangle .
$$

In the limit $N \rightarrow \infty$ we may take

$$
\begin{aligned}
\langle\chi, \chi| h^{-1}\left(t_{n+1}\right) & \mathcal{P} e^{-\int_{t_{n}}^{t_{n+1}} d t A(t)} h\left(t_{n}\right)|\chi, \chi\rangle \\
& \simeq \exp \left(-\left\langle\chi, \chi\left|h^{-1}\left(t_{n}\right) \dot{h}\left(t_{n}\right)+h^{-1}\left(t_{n}\right) A\left(q\left(t_{n}\right)\right) h\left(t_{n}\right)\right| \chi, \chi\right\rangle \Delta t\right) .
\end{aligned}
$$

Using the identity $\langle\chi, \chi|X| \chi, \chi\rangle=i \operatorname{tr}(K X)$ for $X \in \mathfrak{h}$ given in the Appendix, we obtain the coherent state path-integral expession for the holonomy factor ${ }^{3}$

$$
\begin{aligned}
\rho_{\chi \chi}^{\chi}\left(h^{\prime-1}\right. & \left.\mathcal{P} e^{-\int_{0}^{T} d t A} h\right) \\
& =\lim _{N \rightarrow \infty} \int \prod_{n=1}^{N} \widetilde{d h}\left(t_{n}\right) e^{-i \sum_{n} \operatorname{tr} K\left(h^{-1}\left(t_{n}\right) \dot{h}\left(t_{n}\right)+h^{-1}\left(t_{n}\right) A\left(q\left(t_{n}\right)\right) h\left(t_{n}\right)\right) \Delta t} \\
& =\lim _{N \rightarrow \infty} d_{\chi}^{N} V_{S_{K}}^{N} \int_{h}^{h^{\prime}} \mathcal{D} h \delta\left(\xi_{r}\right) \Delta_{\xi} e^{-i \int_{0}^{T} \operatorname{tr}\left(K h^{-1} \dot{h}+h K h^{-1} A(q)\right)} .
\end{aligned}
$$

3 The derivation of the path-integral formula for the holonomy factor (Wilson loop) is also discussed in [22] based on a similar but seemingly different method. 
Inserting (3.21) into (3.16), we obtain the scalar-valued path-integral,

$$
K_{\text {phy }}^{G / H}\left(g^{\prime}, g ; T\right)=\mathcal{N} \int_{q}^{q^{\prime}} \mathcal{D} q \int_{h}^{h^{\prime}} \mathcal{D} h \delta\left(\xi_{r}\right) \Delta_{\xi} e^{i S_{G / H}(q)-i \int_{0}^{T} \operatorname{tr}\left(K h^{-1} h+h K h^{-1} A(q)\right)}
$$

with $\mathcal{N}=e^{-\frac{i}{2} C_{2}^{\chi} T} \lim _{N \rightarrow \infty} d_{\chi}^{N+1} V_{S_{K}}^{N}$, which agrees precisely with the effective pathintegral $^{4} K_{\text {eff }}^{G / H}\left(g^{\prime}, g ; T\right)$ in (3.13). Although, as is well-known [16], derivation of pathintegrals using the (generalized) coherent states remains purely formal, the above argument provides another confirmation (along with the argument by the induced representations given in Section 3.1) in the path-integral framework that the constraint point of view on Mackey's quantization using Dirac's approach is in fact valid.

\section{Conclusion and discussion}

We have seen in this paper that the 'lift and then project' principle leads to a pathintegral which reproduces correctly the two important aspects of the quantum theory on a homogeneous space $G / H$, namely, the inequivalent quantizations and the induced gauge field. The path-integral formula on $G / H$ derived from the principle is given by the pathintegral on $G$ with the $H$ degrees of freedom smeared out with weight factors. Consistency (composition law) requires the weight factors to form a representation of $H$, rendering the entire formula analogous to the familiar one for a multiply connected space $\bar{Q} / \Gamma$ in $[1,4]$. We also derived the holonomy factor in the path-integral after isolating the integration on $G / H$ from that on $H$, and this derivation indicates that the induced gauge field that appears in the holonomy factor is a direct consequence of the weight factors, that is, the essential ingredient of the inequivalent quantizations. The scalar-valued path-integral obtained in the effective Dirac approach $[13,14]$ is then derived from our matrix-valued form using the coherent state path-integral technique.

As mentioned in the Introduction, the fact that at least for the two types of spaces, $Q=G / H$ and $Q=\bar{Q} / \Gamma$, quantum theory is characterized by the irreducible unitary representations of the isometry group, $H$ or $\Gamma$, and not just those of the fundamental group of the space $\pi_{1}(Q)$, suggests that the path-integral formula (2.5) presented here is a generalization of the formula (1.1) for a multiply connected space. Once this interpretation is accepted, it is perhaps natural to extend the path-integral approach further to inhomogeneous spaces. In fact, inhomogeneous spaces often arise in physics, with the one most frequently discussed being a Riemann surface with higher genus. A possible

4 For completeness we mention that in $[13,14]$ the factor $e^{-\frac{i}{2} C_{2}^{\chi} T}$ is dropped during the path-integral reduction on the ground that it merely gives a constant shift in the effective Lagrangian. 
extension to inhomogeneous spaces is the following ${ }^{5}$. Let $P$ be a Riemannian manifold with a metric $g_{P}$ and let a Lie group $H$ act on $P$ freely and isometrically. The manifold $Q=P / H$ then admits an induced metric $g_{Q}$, with which the projection $\pi: P \rightarrow Q$ defines a principal bundle and a Riemannian submersion. Now, given a propagator on $P$ which is $H$-invariant $K_{P}\left(p^{\prime} h, p h ; t\right)=K_{P}\left(p^{\prime}, p ; t\right)$, a straightforward extension of our formulation of the path-integral to such inhomogeneous spaces is possible by defining the propagator on $Q$ as

$$
K^{Q}\left(p^{\prime}, p ; T\right)=\int_{H} d h \rho^{\chi}(h) K^{P}\left(p^{\prime} h, p ; T\right),
$$

which acts on $\chi$-equivariant functions $f: P \rightarrow V_{\chi} ; f(p h)=\rho^{\chi}(h)^{-1} f(p)$.

However, the biggest problem with this extension is that there is no guarantee of having a uniquely defined propagator on $P$ for describing the transition amplitude, because we do not know how to quantize on $P$ in the first place. In fact, the basic reason why we lift our system to the universal covering space $\bar{Q}$ in the case of a multiply connected space, or to a group manifold $G$ in the case of a homogeneous space, is that we know in principle how to quantize on these spaces, and this actually limits the scope of extension of quantization irrespective of the approach employed. Another problem of the extension to inhomogeneous spaces is that we seem to lose any control on the form of the induced connection. This is so because, when the base space $\left(M, g_{M}\right)$ is fixed, inequivalent quantizations depend on the choice of the principal bundle $P$, the lifted metric $g_{P}$ and the representation $\rho^{\chi}$, which amounts to an introduction of an arbitrary connection. This problem does not arise for a homogeneous space, where there exists a criterion to choose a specific connection, since the invariance under the $G$-action determines both $g_{G / H}$ and $g_{G}$ uniquely, leading to the $G$-invariant canonical connection [25]. The existence of such a transitive $G$-action is not only crucial for specifying the connection but also important for the existence of momentum, because a self-adjoint momentum operator can then be defined globally as a generator of the transitive action, which in turn is important in defining a self-adjoint Hamiltonian to provide a unitary time evolution of the system.

If, on the other hand, we have some means to quantize on $P$, then the formula (4.1) may provide a basis not just for quantizing on $Q=P / H$ but for extending even further to field theory, such as the Yang-Mills theory whose configuration space is $\mathcal{A} / \mathcal{G}$, where $\mathcal{A}$ is the space of all gauge potentials and $\mathcal{G}$ the group of gauge transformations. Being an affine space, the space $\mathcal{A}$ seems to admit a conventional quantization and, accordingly, may ensure the extension of our path-integral formula, too. We will not discuss here the

5 This type of construction has already been considered in [23] in investigating geometric properties of induced gauge fields of deformable bodies, and has also been taken up recently in [24] in an attempt to the extension based on the method of geometric quantization. 
outcome of the application of the formula, but a preliminary investigation of the YangMills theory shows that there exist various quantum effects, in addition to the celebrated $\theta$-vacuum structure which is the effect of $\pi_{1}(\mathcal{A} / \mathcal{G})=\mathbb{Z}$, which we have not uncovered yet. We hope to report the full detail of the investigation elsewhere in the near future.

Acknowledgements: S.T. wishes to thank T. Iwai and Y. Uwano for their support in this work, and I.T. is grateful to L. Fehér, D. McMullan and A. Wipf for helpful discussions. This work is supported in part by the Grant-in-Aid for Scientific Research from the Ministry of Education, Science and Culture (No.07804015 and No.08740200).

\section{Appendix. Conventions}

In this appendix we shall provide our conventions on Lie groups/algebras together with some basic facts on coset spaces used in the text (see, for example [18, 26]).

Let $\mathrm{G}$ be a semisimple group and $\mathfrak{g}$ its Lie algebra. In the Lie algebra $\mathfrak{g}$ (or in the complex extension $\mathfrak{g}_{c}$ of $\mathfrak{g}$ ) one can choose the Chevalley basis $\left\{H_{\alpha}, E_{ \pm \varphi}\right\}$ where $\alpha$ are simple roots and $\varphi$ are positive roots. The basis satisfies the relations

$$
\left[E_{\alpha}, E_{-\alpha}\right]=H_{\alpha}, \quad\left[H_{\alpha}, E_{\beta}\right]=K_{\beta \alpha} E_{\beta},
$$

for simple roots $\alpha, \beta$, and $K_{\beta \alpha}=\beta\left(H_{\alpha}\right)=\frac{2 \beta \cdot \alpha}{|\alpha|^{2}}$ is the Cartan matrix. To every dominant weight $\chi$ there exists an irreducible representation — highest weight representation — of $\mathfrak{g}$ in which the Cartan elements $H_{\alpha}$ are diagonal; in particular, on the states $|\chi, \mu\rangle$ specified by the weights $\mu$ connected to the dominant weight $\chi$ (identified as the highest weight in the representation) the eigenvalues are all integer:

$$
H_{\alpha}|\chi, \mu\rangle=\mu\left(H_{\alpha}\right)|\chi, \mu\rangle, \quad \text { with } \quad \mu\left(H_{\alpha}\right)=\frac{2 \mu \cdot \alpha}{|\alpha|^{2}} \in \mathbb{Z} .
$$

On account of this, we can use the dominant weight $\chi$ (or the set of integers $\chi\left(H_{\alpha_{r}}\right)$ for $r=1, \ldots, \operatorname{rank} G$ ) to label the irreducible representation. (To avoid any confusion, we note that in the text the Chevalley basis and the highest weight representations are considered for the subgroup $H$ of $G$, not for $G$.)

Given a closed subgroup $H$ of $G$ with its Lie algebra $\mathfrak{h}$, we take an orthogonal decomposition of $\mathfrak{g}$,

$$
\mathfrak{g}=\mathfrak{h} \oplus \mathfrak{r}
$$

where $\mathfrak{r}=\mathfrak{h}^{\perp}$ is the orthogonal complement of $\mathfrak{h}$ to $g$ with respect to the innerproduct $(\mathfrak{h}, \mathfrak{r})=0$ which is defined by the trace in an irreducible representation $\rho ;(X, Y)=$ 
$\operatorname{tr}(\rho(X) \rho(Y))$ for $X, Y \in \mathfrak{g}$. (We often omit the $\rho$ in the trace and write it simply as $\operatorname{tr}(X Y)$.) The decomposition (A.3) is, in fact, reductive, i.e., $[\mathfrak{h}, \mathfrak{r}] \subset \mathfrak{r}$, because $[\mathfrak{h}, \mathfrak{h}] \subset \mathfrak{h}$ and the orthogonality imply $0=([\mathfrak{h}, \mathfrak{h}], \mathfrak{r})=(\mathfrak{h},[\mathfrak{h}, \mathfrak{r}])$. The decomposition of an element $X \in \mathfrak{g}$ according to (A.3) is written as $X=\left.X\right|_{\mathfrak{h}}+\left.X\right|_{\mathfrak{r}}$. We shall denote bases of the spaces by

$$
\begin{aligned}
\mathfrak{g} & =\operatorname{span}\left\{T_{m}\right\}, & m & =1, \ldots, \operatorname{dim} G, \\
\mathfrak{h} & =\operatorname{span}\left\{T_{i}\right\}, & i & =1, \ldots, \operatorname{dim} H, \\
\mathfrak{r} & =\operatorname{span}\left\{T_{a}\right\}, \quad & a & =1, \ldots, \operatorname{dim}(G / H) .
\end{aligned}
$$

Using a properly normalized trace one has $\left(T_{m}, T_{n}\right)=\delta_{m n}$.

Choosing in particular the Chevalley basis $\left\{H_{\alpha}, E_{ \pm \varphi}\right\}$ for our basis in $\mathfrak{g}$, we can expand any anti-Hermitian element, $X \in \mathfrak{g}$ with $X^{\dagger}=-X$, as

$$
X=\sum_{\alpha} X_{\alpha} \frac{1}{i} H_{\alpha}+\sum_{ \pm \varphi} X_{ \pm \varphi} E_{ \pm \varphi},
$$

where $X_{\alpha}, X_{ \pm \varphi}$ are the coefficients of the expansion. Let $K$ be the element belonging to the Cartan subalgebra of $\mathfrak{g}$ carrying the label of the representation $\chi$ in the coefficients such that $\left(\frac{1}{i} H_{\alpha}, K\right)=\chi\left(H_{\alpha}\right)$. Then we have the identity

$$
\langle\chi, \chi|X| \chi, \chi\rangle=i(K, X) .
$$

We can furnish a Riemannian metric on the coset space $G / H$ as follows. Decompose first $g \in \mathrm{G}$ as $g=\sigma h$ where $\sigma \in G$ is a (local) section and $h \in H$. Then form the Maurer-Cartan 1-form $\sigma^{-1} d \sigma$ and split it as $\sigma^{-1} d \sigma=\left.\sigma^{-1} d \sigma\right|_{\mathfrak{h}}+\left.\sigma^{-1} d \sigma\right|_{\mathfrak{r}}$, where the first part $\left.\sigma^{-1} d \sigma\right|_{\mathfrak{h}}$ leads to the canonical connection $A$ mentioned in the text. The second part $e:=\left.\sigma^{-1} d \sigma\right|_{\mathfrak{r}}$, on the other hand, is regarded as a vielbein, from which the metric on $G / H$ is defined by

$$
d s^{2}=g_{\alpha \beta} d q^{\alpha} \otimes d q^{\beta}:=\operatorname{tr}(e \otimes e),
$$

where $\left\{q^{\alpha}\right\}, \alpha=1, \ldots, n(n=\operatorname{dim}(G / H))$, is a set of local coordinates on $G / H$. 


\section{References}

[1] M.G.G. Laidlaw and C.M. DeWitt, Phys. Rev. D3 (1971) 1375.

[2] L.S. Schulman, Phys. Rev. 176 (1968) 1558.

[3] L.S. Schulman, "Techniques and Applications of Path Integration", Willey, New York, 1981.

[4] J.S. Dowker, J. Phys. A5 (1972) 936.

[5] P.A. Horvathy, G. Morandi and E.C.G. Sudarshan, Nuovo Cimento 11D (1989) 201.

[6] G.W. Mackey, "Induced Representations of Groups and Quantum Mechanics", Benjamin, New York, 1969.

[7] C.J. Isham, in "Relativity, Groups and Topology II", (B.S. DeWitt and R. Stora, Eds) North-Holland, Amsterdam, 1984.

[8] N.P. Landsman and N. Linden, Nucl. Phys. B365 (1991) 121.

[9] S. Kobayashi and K. Nomizu, "Foundations of Differential Geometry" Vols.I,II (Interscience, New York, 1969).

[10] F.A. Bais and P. Batenburg, Nucl. Phys. B269 (1986) 363.

[11] Y. Ohnuki and S. Kitakado, Mod. Phys. Lett. A7 (1992) 2477; J. Math. Phys. 34 (1993) 2827.

[12] S. Tanimura and I. Tsutsui, Mod. Phys. Lett. A34 (1995) 2607.

[13] D. McMullan and I. Tsutsui, Phys. Lett. 320B (1994) 287.

[14] D. McMullan and I. Tsutsui, Ann. Phys. 237 (1995) 269.

[15] P.A.M. Dirac, "Lectures on Quantum Mechanics", Yeshiva, New York, 1964.

[16] J.R. Klauder and B.-S. Skagerstam (Eds.), "Coherent States", World Scientific, Singapore, 1985.

[17] Y. Aharonov and D. Bohm, Phys. Rev. 115 (1959) 485.

[18] S.E. Humphreys, "Introduction to Lie Algebras and Representation Theory", Springer-Verlag, Berlin, 1972.

[19] R. Abraham and J.E. Marsden, "Foundations of Mechanics", Second Edition, Benjamin/Cummings, Reading, 1978.

[20] A.M. Perelomov, "Integrable Systems of Classical Mechanics and Lie Algebras", Birkhäuser Verlag, Basel, 1990. 
[21] S.K. Wong, Nuovo Cimento 65A (1970), 689.

[22] D.I. Diakonov and V.Yu. Petrov, Phys. Lett. 224B (1989) 131.

[23] R. Montgomery, Commun. Math. Phys. 128 (1990) 565.

[24] M. A. Robson, Phys. Lett. 335B (1994) 383; J. Geom. Phys. 19 (1996) 207.

[25] P. Lévay, D. McMullan and I. Tsutsui, J. Math. Phys. 37 (1996) 625.

[26] A.O. Barut and R. Raczka, "Theory of Group Representations and Applications", Polish Scientific Publishers, Warszawa, 1977. 